\title{
A PROPERTY OF ALGEBRAIC UNIVOQUE NUMBERS
}

\author{
M. DE VRIES* \\ Delft University of Technology, Mekelweg 4, 2628 CD Delft, the Netherlands \\ e-mail: w.m.devries@ewi.tudelft.nl \\ (Received December 18, 2006; accepted February 1, 2007)
}

\begin{abstract}
Consider the set $\mathcal{U}$ of real numbers $q \geqq 1$ for which only one sequence $\left(c_{i}\right)$ of integers $0 \leqq c_{i} \leqq q$ satisfies the equality $\sum_{i=1}^{\infty} c_{i} q^{-i}=1$. We show that the set of algebraic numbers in $\mathcal{U}$ is dense in the closure $\overline{\mathcal{U}}$ of $\mathcal{U}$.
\end{abstract}

\section{Introduction}

Given a real number $q \geqq 1$, a $q$-expansion (or simply expansion) is a sequence $\left(c_{i}\right)=c_{1} c_{2} \ldots$ of integers satisfying $0 \leqq c_{i} \leqq q$ for all $i \geqq 1$ such that

$$
\frac{c_{1}}{q}+\frac{c_{2}}{q^{2}}+\frac{c_{3}}{q^{3}}+\cdots=1
$$

The greedy expansion, denoted by $\left(\gamma_{i}(q)\right)=\left(\gamma_{i}\right)$, is obtained by performing the greedy algorithm of Rényi [11]: if $\gamma_{i}$ is already defined for $i<n$, then $\gamma_{n}$ is the largest integer satisfying

$$
\sum_{i=1}^{n} \frac{\gamma_{i}}{q^{i}} \leqq 1 .
$$

*The author has been supported by NWO, Project nr. ISK04G.

Key words and phrases: beta-expansions, univoque numbers.

2000 Mathematics Subject Classification: primary: 11A63, secondary: 11B83.

0236-5294/\$20.00 C 2007 Akadémiai Kiadó, Budapest 
Equivalently, $\left(\gamma_{i}\right)$ is the largest expansion in lexicographical order.

If $q>1$, then quasi-greedy expansion, denoted by $\left(\alpha_{i}(q)\right)=\left(\alpha_{i}\right)$, is obtained by performing the quasi-greedy algorithm: if $\alpha_{i}$ is already defined for $i<n$, then $\alpha_{n}$ is the largest integer satisfying

$$
\sum_{i=1}^{n} \frac{\alpha_{i}}{q^{i}}<1 .
$$

An expansion is called infinite if it contains infinitely many nonzero terms; otherwise it is called finite. Observe that there are no infinite expansions if $q=1$ : the only 1 -expansions are given by $1(0)^{\infty}, 01(0)^{\infty}, 001(0)^{\infty}, \ldots$ On the other hand, if $q>1$, then $\left(\alpha_{i}\right)$ is the largest infinite expansion in lexicographical order.

For any given $q>1$, the following relations between the quasi-greedy expansion and the greedy expansion are straightforward. The greedy expansion is finite if and only if $\left(\alpha_{i}\right)$ is periodic. If $\left(\gamma_{i}\right)$ is finite and $\gamma_{m}$ is its last nonzero term, then $m$ is the smallest period of $\left(\alpha_{i}\right)$, and

$$
\alpha_{i}=\gamma_{i} \quad \text { for } \quad i=1, \ldots, m-1, \quad \text { and } \quad \alpha_{m}=\gamma_{m}-1 \text {. }
$$

Erdôs, Horváth and Joó [4] discovered that for some real numbers $q>1$ there exists only one $q$-expansion. Subsequently, the set $\mathcal{U}$ of such univoque numbers was characterized in [5], [6], [9] (see Theorem 2.1). Using this characterization, Komornik and Loreti showed in [7] that $\mathcal{U}$ has a smallest element $q^{\prime} \approx 1.787$ and the corresponding expansion $\left(\tau_{i}\right)$ is given by the truncated Thue-Morse sequence, defined by setting $\tau_{2^{N}}=1$ for $N=0,1, \ldots$ and

$$
\tau_{2^{N}+i}=1-\tau_{i} \quad \text { for } \quad 1 \leqq i<2^{N}, \quad N=1,2, \ldots
$$

Allouche and Cosnard [1] proved that the number $q^{\prime}$ is transcendental. This raised the question whether there exists a smallest algebraic univoque number. Komornik, Loreti and Pethó [8] answered this question in the negative by constructing a decreasing sequence $\left(q_{n}\right)$ of algebraic univoque numbers converging to $q^{\prime}$.

It is the aim of this note to show that for each $q \in \mathcal{U}$ there exists a sequence of algebraic univoque numbers converging to $q$ :

THEOREM 1.1. The set $\mathcal{A}$ consisting of all algebraic univoque numbers is dense in $\overline{\mathcal{U}}$.

Our proof of Theorem 1.1 relies on a characterization of the closure $\overline{\mathcal{U}}$ of $\mathcal{U}$, recently obtained by Komornik and Loreti in [9] (see Theorem 2.2). 


\section{Proof of Theorem 1.1}

In the sequel, a sequence always means a sequence of nonnegative integers. We use systematically the lexicographical order between sequences; we write $\left(a_{i}\right)<\left(b_{i}\right)$ if there exists an index $n \geqq 1$ such that $a_{i}=b_{i}$ for $i<n$ and $a_{n}<b_{n}$. This definition extends in the obvious way to sequences of finite length.

The following algebraic characterization of the set $\mathcal{U}$ can be found in [5], [6], [9]:

THEOREM 2.1. The map $q \mapsto\left(\gamma_{i}(q)\right)$ is a strictly increasing bijection between the set $\mathcal{U}$ and the set of all sequences $\left(\gamma_{i}\right)$ satisfying

$$
\gamma_{j+1} \gamma_{j+2} \ldots<\gamma_{1} \gamma_{2} \ldots \text { for all } j \geqq 1
$$

and

$$
\overline{\gamma_{j+1} \gamma_{j+2} \ldots}<\gamma_{1} \gamma_{2} \ldots \text { for all } j \geqq 1
$$

where we use the notation $\overline{\gamma_{n}}:=\gamma_{1}-\gamma_{n}$.

REMARK. It was essentially shown by Parry [10] that a sequence $\left(\gamma_{i}\right)$ is the greedy $q$-expansion for some $q \geqq 1$ if and only if $\left(\gamma_{i}\right)$ satisfies the condition (2.1).

Using the above result, Komornik and Loreti [9] investigated the topological structure of the set $\mathcal{U}$. In particular they showed that $\overline{\mathcal{U}} \backslash \mathcal{U}$ is dense in $\overline{\mathcal{U}}$. Hence the set $\overline{\mathcal{U}}$ is a perfect set. Moreover, they established an analogous characterization of the closure $\overline{\mathcal{U}}$ of $\mathcal{U}$ :

THEOREM 2.2. The map $q \mapsto\left(\alpha_{i}(q)\right)$ is a strictly increasing bijection between the set $\overline{\mathcal{U}}$ and the set of all sequences $\left(\alpha_{i}\right)$ satisfying

$$
\alpha_{j+1} \alpha_{j+2} \ldots \leqq \alpha_{1} \alpha_{2} \ldots \text { for all } j \geqq 1
$$

and

$$
\overline{\alpha_{j+1} \alpha_{j+2} \ldots}<\alpha_{1} \alpha_{2} \ldots \text { for all } j \geqq 1
$$

where we use the notation $\overline{\alpha_{n}}:=\alpha_{1}-\alpha_{n}$.

REMARKS. 1. It was shown in [3] that a sequence $\left(\alpha_{i}\right)$ is the quasi-greedy $q$-expansion for some $q>1$ if and only if $\left(\alpha_{i}\right)$ is infinite and satisfies (2.3). Note also that a sequence satisfying (2.3) and (2.4) is automatically infinite.

2. If $q \in \overline{\mathcal{U}} \backslash \mathcal{U}$, then we must have equality in (2.3) for some $j \geqq 1$, i.e., the greedy $q$-expansion is finite for each $q \in \overline{\mathcal{U}} \backslash \mathcal{U}$. On the other hand, it follows from Theorems 2.1 and 2.2 that a sequence of the form $\left(1^{n} 0\right)^{\infty}(n \geqq 2)$ is the quasi-greedy $q$-expansion for some $q \in \overline{\mathcal{U}} \backslash \mathcal{U}$. Hence the set $\overline{\mathcal{U}} \backslash \mathcal{U}$ is countably infinite. 
The following technical lemma is a direct consequence of Theorem 2.2 and Lemmas 3.4 and 4.1 in [9]:

LEMMA 2.3. Let $\left(\alpha_{i}\right)$ be a sequence satisfying (2.3) and (2.4). Then

(i) there exist arbitrary large integers $m$ such that

$$
\overline{\alpha_{j+1} \ldots \alpha_{m}}<\alpha_{1} \ldots \alpha_{m-j} \quad \text { for all } 0 \leqq j<m
$$

(ii) for all positive integers $m \geqq 1$,

$$
\overline{\alpha_{1} \ldots \alpha_{m}}<\alpha_{m+1} \ldots \alpha_{2 m} .
$$

Proof of Theorem 1.1. Since the set $\overline{\mathcal{U}} \backslash \mathcal{U}$ is dense in $\overline{\mathcal{U}}$, it is sufficient to show that $\overline{\mathcal{A}} \supset \overline{\mathcal{U}} \backslash \mathcal{U}$. In order to do so, fix $q \in \overline{\mathcal{U}} \backslash \mathcal{U}$. Then, according to Theorem 2.2 , the quasi-greedy $q$-expansion $\left(\alpha_{i}\right)$ satisfies (2.3) and (2.4). Let $k$ be a positive integer for which equality holds in (2.3), i.e., $\left(\alpha_{i}\right)=\left(\alpha_{1} \ldots \alpha_{k}\right)^{\infty}$. According to Lemma 2.3 there exists an integer $m \geqq k$ such that (2.5) is satisfied. Let $N$ be a positive integer such that $k N \geqq m$ and consider the sequence

$$
\left(\gamma_{i}\right)=\left(\gamma_{i}^{N}\right)=\left(\alpha_{1} \ldots \alpha_{k}\right)^{N}\left(\alpha_{1} \ldots \alpha_{m} \overline{\alpha_{1} \ldots \alpha_{m}}\right)^{\infty} .
$$

For ease of exposition we suppress the dependence of $\left(\gamma_{i}\right)$ on $N$. Note that $\gamma_{i}=\alpha_{i}$ for $1 \leqq i \leqq m+k N$. In particular, we have

$$
\gamma_{i}=\alpha_{i} \text { for } 1 \leqq i \leqq 2 m .
$$

Since $\left(\gamma_{i}\right)$ has a periodic tail, the number $q_{N}$ determined by

$$
1=\sum_{i=1}^{\infty} \frac{\gamma_{i}}{q_{N}^{i}}
$$

is an algebraic number and $q_{N} \rightarrow q$ as $N \rightarrow \infty$.

According to Theorem 2.1 it remains to verify the inequalities (2.1) and (2.2). First we verify $(2.1)$ and $(2.2)$ for $j \geqq k N$. For those values of $j$ the inequality (2.1) for $j+m$ is equivalent to (2.2) for $j$ and (2.2) for $j+m$ is equivalent to (2.1) for $j$. Therefore it suffices to verify the inequalities (2.1) and (2.2) for $k N \leqq j<k N+m$. Fix $k N \leqq j<k N+m$. From (2.3), (2.6) and (2.7) we have

$$
\begin{gathered}
\gamma_{j+1} \ldots \gamma_{k N+2 m}=\alpha_{j-k N+1} \ldots \alpha_{m} \overline{\alpha_{1} \ldots \alpha_{m}} \\
<\alpha_{j-k N+1} \ldots \alpha_{m} \alpha_{m+1} \ldots \alpha_{2 m} \leqq \alpha_{1} \ldots \alpha_{k N+2 m-j}=\gamma_{1} \ldots \gamma_{k N+2 m-j}
\end{gathered}
$$


and from inequality (2.5) we have

$$
\overline{\gamma_{j+1} \ldots \gamma_{k N+m}}=\overline{\alpha_{j-k N+1} \ldots \alpha_{m}}<\alpha_{1} \ldots \alpha_{k N+m-j}=\gamma_{1} \ldots \gamma_{k N+m-j} .
$$

Now we verify (2.1) for $j<k N$. If $m \leqq j<k N$, then by (2.3) and (2.6),

$$
\gamma_{j+1} \ldots \gamma_{k N+2 m}<\alpha_{j+1} \ldots \alpha_{k N+2 m} \leqq \alpha_{1} \ldots \alpha_{k N+2 m-j}=\gamma_{1} \ldots \gamma_{k N+2 m-j} .
$$

If $1 \leqq j<m$, then by (2.3) and (2.5),

$$
\begin{gathered}
\gamma_{j+1} \ldots \gamma_{k N+m+j}=\alpha_{j+1} \ldots \alpha_{k N+m} \overline{\alpha_{1} \ldots \alpha_{j}} \\
\leqq \alpha_{1} \ldots \alpha_{k N+m-j} \overline{\alpha_{1} \ldots \alpha_{j}}<\alpha_{1} \ldots \alpha_{k N+m-j} \alpha_{m-j+1} \ldots \alpha_{m}=\gamma_{1} \ldots \gamma_{k N+m} .
\end{gathered}
$$

Finally, we verify (2.2) for $j<k N$. Write $j=k \ell+i, 0 \leqq \ell<N$ and $0 \leqq i$ $<k$. If $i=0$, then $(2.2)$ follows from the relation

$$
\overline{\gamma_{j+1}}=\overline{\alpha_{1}}=0<\alpha_{1}=\gamma_{1} .
$$

If $1 \leqq i<k$, then applying Lemma $2.3\left(\right.$ ii) we get $\overline{\alpha_{i+1} \ldots \alpha_{2 i}}<\alpha_{1} \ldots \alpha_{i}$. Hence

$$
\overline{\gamma_{j+1} \ldots \gamma_{j+k}}=\overline{\alpha_{j+1} \ldots \alpha_{j+k}}=\overline{\alpha_{i+1} \ldots \alpha_{i+k}}<\alpha_{1} \ldots \alpha_{k}=\gamma_{1} \ldots \gamma_{k} .
$$

(In order for the first equality to hold in case $\ell=N-1$, we need the condition $m \geqq k$.)

REMARKS. 1. Since the set $\overline{\mathcal{U}}$ is a perfect set and $\overline{\mathcal{U}} \backslash \mathcal{U}$ is countable, each neighborhood of $q \in \mathcal{U}$ contains uncountably many elements of $\mathcal{U}$. Hence the set of transcendental univoque numbers is dense in $\overline{\mathcal{U}}$ as well.

2. Recently, Allouche, Frougny and Hare [2] proved that there also exist univoque Pisot numbers. In particular they determined the smallest three univoque Pisot numbers.

\section{References}

[1] J.-P. Allouche and M. Cosnard, The Komornik-Loreti constant is transcendental, Amer. Math. Monthly, 107 (2000), 448-449.

[2] J.-P. Allouche, C. Frougny and K. G. Hare, On univoque Pisot numbers, Mathematics of Computation, to appear.

[3] C. Baiocchi and V. Komornik, Quasi-greedy expansions and lazy expansions in noninteger bases, manuscript.

[4] P. Erdós, M. Horváth and I. Joó, On the uniqueness of the expansions $1=\sum q^{-n_{i}}$, Acta Math. Hungar., 58 (1991), 333-342. 
[5] P. Erdős, I. Joó and V. Komornik, Characterization of the unique expansions $1=\sum q^{-n_{i}}$ and related problems, Bull. Soc. Math. France, $118(1990), 377-$ 390.

[6] P. Erdôs, I. Joó and V. Komornik, On the number of q-expansions, Ann. Univ. Sci. Budapest. Eötvös Sect. Math., 37 (1994), 109-118.

[7] V. Komornik and P. Loreti, Unique developments in non-integer bases, Amer. Math. Monthly, 105 (1998), 636-639.

[8] V. Komornik, P. Loreti and A. Pethó, The smallest univoque number is not isolated, Publ. Math. Debrecen, 62 (2003), 429-435.

[9] V. Komornik and P. Loreti, On the topological structure of univoque sets, J. Number Theory, 122 (2007), 157-183.

[10] W. Parry, On the $\beta$-expansions of real numbers, Acta Math. Acad. Sci. Hungar., 11 (1960), 401-416.

[11] A. Rényi, Representations for real numbers and their ergodic properties, Acta Math. Acad. Sci. Hungar., 8 (1957), 477-493. 